\title{
Excitation of Magnetic Dipole Transitions at Optical Frequencies
}

\author{
Mark Kasperczyk, ${ }^{1}$ Steven Person, ${ }^{2}$ Duarte Ananias, ${ }^{3}$ Luis D. Carlos, ${ }^{4}$ and Lukas Novotny ${ }^{1, *}$ \\ ${ }^{1}$ Photonics Laboratory, ETH Zürich, 8093 Zürich, Switzerland \\ ${ }^{2}$ Institute of Optics, University of Rochester, Rochester, New York 14627, USA \\ ${ }^{3}$ Department of Chemistry, CICECO, University of Aveiro, 3810193 Aveiro, Portugal \\ ${ }^{4}$ Department of Physics, CICECO, University of Aveiro, 3810193 Aveiro, Portugal
}

(Received 20 November 2014; published 23 April 2015)

\begin{abstract}
We use the magnetic field distribution of an azimuthally polarized focused laser beam to excite a magnetic dipole transition in $\mathrm{Eu}^{3+}$ ions embedded in a $\mathrm{Y}_{2} \mathrm{O}_{3}$ nanoparticle. The absence of the electric field at the focus of an azimuthally polarized beam allows us to unambiguously demonstrate that the nanoparticle is excited by the magnetic dipole transition near $527.5 \mathrm{~nm}$. When the laser wavelength is resonant with the magnetic dipole transition, the nanoparticle maps the local magnetic field distribution, whereas when the laser wavelength is resonant with an electric dipole transition, the nanoparticle is sensitive to the local electric field. Hence, by tuning the excitation wavelength, we can selectively excite magnetic or electric dipole transitions through optical fields.
\end{abstract}

In the optical frequency regime, magnetic dipole transitions are orders of magnitude weaker than their electric dipole counterparts [1-3]. Because of this, magnetic dipole (MD) transitions are often neglected in optics, and the study of light-matter interactions becomes instead the study of interactions between electric fields and electric dipoles (ED). Perhaps the most well-known exceptions occur in the fields of metamaterials [4] and photonic crystal cavities $[5,6]$, in which specially engineered structures can be produced to enhance interactions with the magnetic field. Nature, however, also provides materials with strong MD transitions, namely, rare earth ions. Many of their MD transitions are found within the visible spectrum, making them promising candidates for the optical excitation of MD transitions.

Much theoretical and experimental work has been done exploring the MD and ED contributions to spontaneous emission from $\mathrm{Eu}^{3+}$ and other trivalent rare earth ions [1,7-10]. Lifetimes and oscillator strengths have been studied as a function of local environment [11-14], ion concentration [15-17], and particle size [18-23]. But so far, research has focused solely on detecting and enhancing spontaneous MD emission, with no work done on selective excitation through magnetic fields. In 1939, Deutschbein first identified the MD character of the ${ }^{7} \mathrm{~F}_{0} \rightarrow{ }^{5} \mathrm{D}_{1}$ transition in $\mathrm{Eu}^{3+}$ (c.f. Fig. 1(a)) by exploiting the birefringence of $\mathrm{Eu}\left(\mathrm{BrO}_{3}\right)_{3} \cdot 9 \mathrm{H}_{2} \mathrm{O}$ and $\mathrm{Eu}\left(\mathrm{C}_{2} \mathrm{H}_{5} \mathrm{SO}_{4}\right)_{3} \cdot 9 \mathrm{H}_{2} \mathrm{O}$ crystals [24]. He could deduce the MD or ED character of a transition by recording absorption or emission spectra for ordinary and extraordinary polarizations and comparing them to a spectrum taken along the $c$ axis of the crystal. However, he could not selectively address individual transitions. Here, we report the direct and selective optical excitation of a MD transition in the rare earth ion $\mathrm{Eu}^{3+}$.
The light-matter interaction between a charge-neutral quantum system and an electromagnetic field can be represented by a multipole expansion of the interaction Hamiltonian

$$
\mathrm{H}_{\mathrm{int}}=-\mathbf{p} \cdot \mathbf{E}(t)-\mathbf{m} \cdot \mathbf{B}(t)-[\mathbf{Q} \nabla] \cdot \mathbf{E}(t)-\cdots,
$$

with $\mathbf{p}$ being the electric dipole moment, $\mathbf{m}$ the magnetic dipole moment, and $\mathbf{Q}$ the electric quadrupole moment (a tensor). The different terms of the interaction Hamiltonian lead to different selection rules, and their magnitudes depend on the size of the quantum system. For an atomic system, characterized by the Bohr radius $a_{0}$, the magnitude of the $\mathrm{ED}$ is $p=e a_{0}$ ( $e$ is the elementary charge) and the magnitude of the MD corresponds to the Bohr magneton (a)

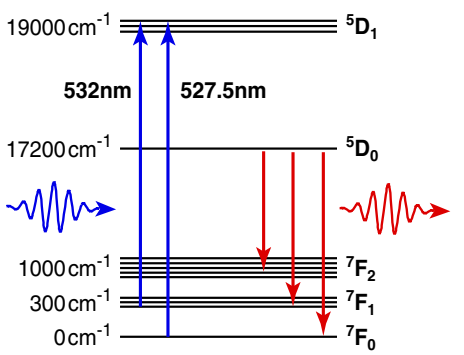

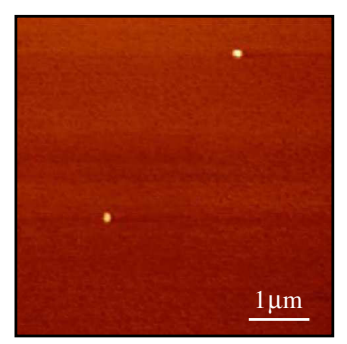

FIG. 1 (color online). (a) Simplified energy level structure of $\mathrm{Eu}^{3+}$ with approximate energy scale on the left hand side. The excitation wavelength (blue arrows) is tuned to be resonant with either the ${ }^{7} \mathrm{~F}_{0} \rightarrow{ }^{5} \mathrm{D}_{1}$ magnetic dipole transition at $527.5 \mathrm{~nm}$ or the ${ }^{7} \mathrm{~F}_{1} \rightarrow{ }^{5} \mathrm{D}_{1}$ electric dipole transition at $532 \mathrm{~nm}$. The emitted signal (red arrows) consists of several different transitions, primarily ${ }^{5} \mathrm{D}_{0} \rightarrow{ }^{7} \mathrm{~F}_{2}$ and ${ }^{5} \mathrm{D}_{0} \rightarrow{ }^{7} \mathrm{~F}_{1}$. (b) Topographic image taken with an atomic force microscope showing two spatially separated $60 \mathrm{~nm} \mathrm{Eu}^{3+}$ nanoparticles. 

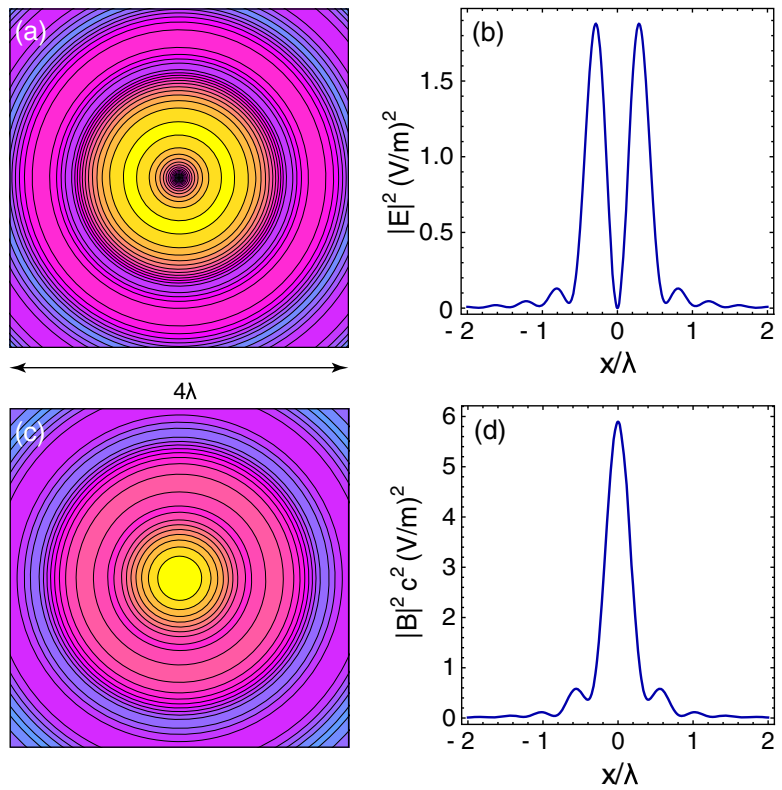

FIG. 2 (color online). Field distribution in the focal plane of an azimuthally polarized laser beam focused on a glass air interface (1.3 NA, filling factor $f_{0}=1$ ). (a) Contours of $|E|^{2}$ (factor of $2^{1 / 2}$ between adjacent lines). (b) Linecut of $|E|^{2}$ showing that the electric field intensity is zero on the optical axis. (c) Contours of $|B|^{2}$ (factor of $2^{1 / 2}$ between adjacent lines). (d) Linecut of $|B|^{2}$ showing that the magnetic field intensity is maximum on the optical axis. Note that the maximum magnetic field intensity in (d) is about 3 times higher than the maximum electric field intensity in (b). All fields are evaluated just above the glass air interface.

$m=\hbar e / 2 m$ ( $m$ is the electron mass). Furthermore, the magnetic field of a plane wave in free space is $B=E / c$ ( $c$ is the speed of light), and hence, the MD interaction is a factor $\hbar /\left(m c a_{0}\right)=1 / 137=\alpha$ weaker than the ED interaction. To measure MD interactions, we require a large quantum system (replacing $a_{0}$ ), ED forbidden transitions, and/or a zero electric field. Typically, the selection rules of the electric quadrupole interaction [third term in Eq. (1)] are identical to those of the MD interaction [25] and therefore we require a zero electric field to unambiguously excite a MD transition. Such a situation can be achieved with an azimuthally polarized laser beam $[26,27]$ as used in this work.

In our experiments we use $60 \mathrm{~nm}$ particles of $\mathrm{Y}_{2} \mathrm{O}_{3}$ doped with $\mathrm{Eu}^{3+}$ ions. The $\mathrm{Eu}^{3+}$ concentration is $10^{22}$ ions $/ \mathrm{cm}^{3}$ (38\% mol). Figure 1(a) shows the energy level structure of $\mathrm{Eu}^{3+}$. We prepared the particles as a powder through a room temperature colloidal coprecipitation method developed by Gowd et al. [28]. The powder is then dissolved in water, ultrasonicated, and deposited on a glass coverslip by spin coating, resulting in spatially separated $\mathrm{Eu}^{3+}$ nanocrystals [see Fig. 1(b)]. Prefabricated markers on the coverslip make it possible to locate the same particle in several different experiments.
Individual $\mathrm{Eu}^{3+}$ nanoparticles are then excited by an azimuthally polarized laser beam. The electric field intensity in the focal plane of a strongly focused azimuthally polarized beam forms a ring, or doughnut, around the optical axis, with an intensity null on axis. Figures 2(a) and 2(b) show the characteristic doughnut pattern of a tightly focused azimuthal beam together with a horizontal linecut through the center of the doughnut, demonstrating that the on-axis intensity of the electric field is equal to zero. The magnetic field of this beam, however, shows a different pattern. As seen in Figs. 2(c) and 2(d), the magnetic field distribution forms a circular bright spot, with maximum intensity on axis. Raster scanning a single $\mathrm{Eu}^{3+}$ nanoparticle through the focus will map either the magnetic or the electric field intensity distribution, depending on whether the particle exhibits a MD or ED transition at the wavelength of the excitation beam. Since an azimuthally polarized beam has different magnetic and electric focal field distributions, it is straightforward to determine whether the nanoparticle is interacting with the electric or magnetic field, based on the fluorescence image formed when raster scanning the nanoparticle through the stationary excitation beam.

As illustrated in Fig. 3(a), single nanoparticles are excited by the focused light $(\mathrm{NA}=1.3)$ from a tunable optical parametric oscillator (OPO). Before entering the objective lens, the OPO laser is converted to an azimuthally polarized beam by sending it through a liquid crystal polarization converter and then through a spatial filter. Spontaneous emission from single nanocrystals is collected using the same objective lens. A dichroic beam splitter (not shown) separates the collected signal from the excitation laser, and bandpass filters block any remaining light at the
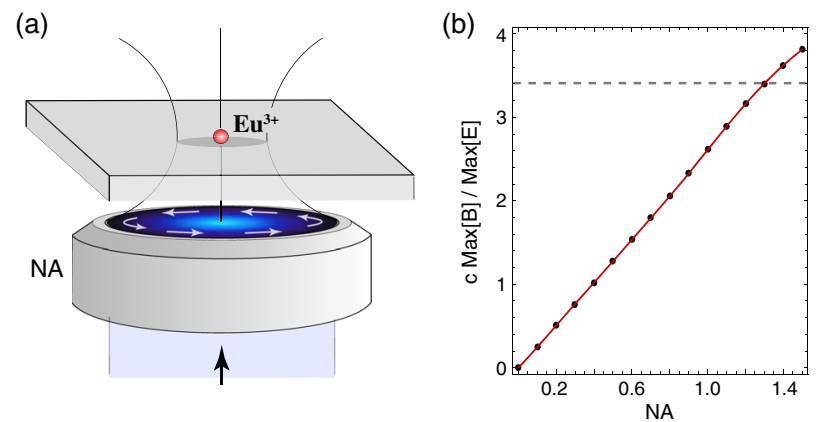

FIG. 3 (color online). (a) Illustration of the experiment. A spatially isolated $\mathrm{Eu}^{3+}: \mathrm{Y}_{2} \mathrm{O}_{3}$ nanoparticle is excited by an azimuthally polarized beam (the arrows show the direction of electric field vector) focused by a 1.3 NA objective lens. The same lens is used to collect spontaneously emitted photons from the nanoparticle. (b) Ratio of the maximum magnetic field strength (center of beam) to the maximum electric field strength (outer ring) of an azimuthally polarized laser beam as a function of the numerical aperture. The field strengths were evaluated just above a glass air interface. The horizontal dashed line indicates the ratio for $\mathrm{NA}=1.3$ used in our experiments. 

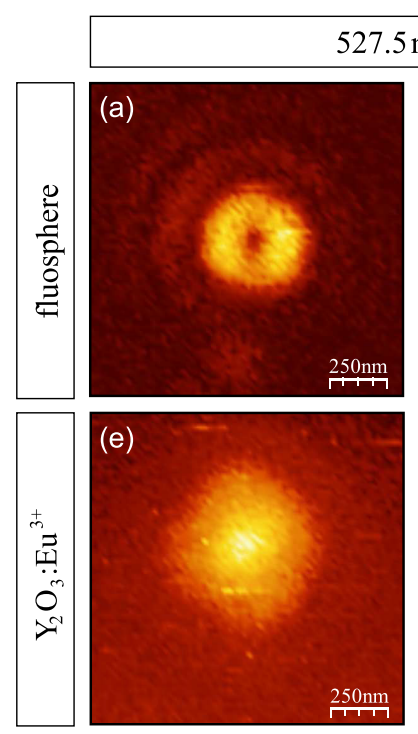
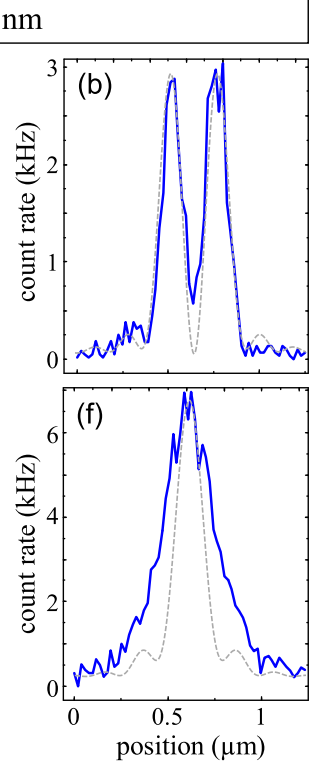
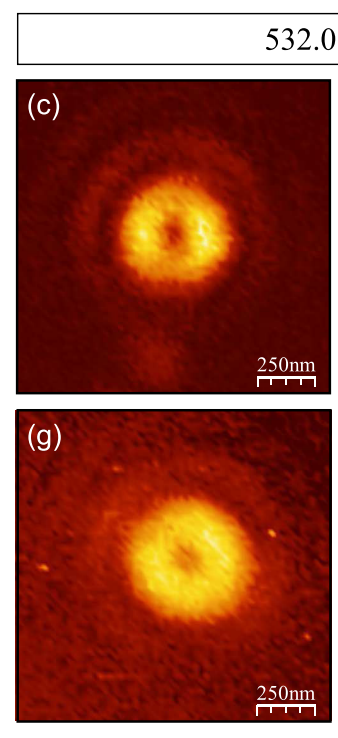
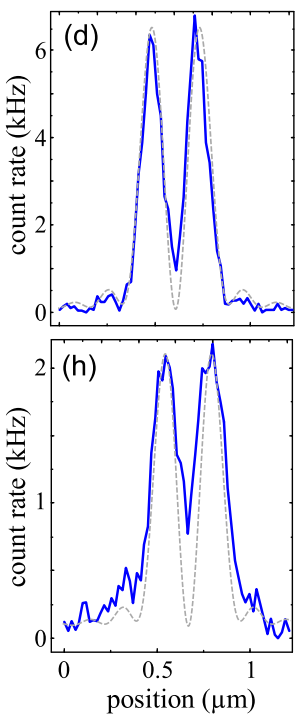

FIG. 4 (color online). Excitation rate images of an $\mathrm{Eu}^{3+}$ nanocrystal and a dye doped nanoparticle (fluosphere). (a) Scan of $20 \mathrm{~nm}$ fluosphere excited with an azimuthally polarized beam with wavelength $527.5 \mathrm{~nm}$. The dye molecules are excited through an electric dipole transition and hence the image renders the electric field distribution of the excitation beam. (b) Linecut showing the intensity dip in the center of the beam. (c) Scan of the same fluosphere, but with the excitation wavelength tuned to $532 \mathrm{~nm}$. (d) Linecut showing the intensity dip in the center of the beam. (e) Scan of $60 \mathrm{~nm} \mathrm{Y} \mathrm{O}_{3}: \mathrm{Eu}^{3+}$ nanoparticle excited with an azimuthally polarized beam with wavelength $527.5 \mathrm{~nm}$. Since $\mathrm{Eu}^{3+}$ ions exhibit a MD transition at $527.5 \mathrm{~nm}$, the image provides a map of the magnetic field distribution [cf. Fig. 2(c)]. (f) Linecut showing that the signal is maximum at the center. (g) Scan of the same $\mathrm{Y}_{2} \mathrm{O}_{3}$ : Eu ${ }^{3+}$, but with the excitation wavelength tuned to $532 \mathrm{~nm}$. At this wavelength, Eu ${ }^{3+}$ ions exhibit an ED transition, and hence the image is a map of the electric field distribution. (h) Linecut showing an intensity minimum at the center. The experimental cross sections (b),(d),(f),(h) are overlaid with theoretical cross sections (dashed curves) from Figs. 2(b) and 2(d), reproduced here for ease of comparison. The deviation between theory and experiment in (f) is due to off center ED excitations (see Ref. [30]).

laser line. The signal is detected using an avalanche photodiode or a spectrometer. Excitation-rate images are recorded by raster scanning a selected nanoparticle in the focal plane of the excitation beam and measuring the fluorescence intensity pixel by pixel. The detected signal arises from a combination of ED and MD transitions at lower energy than the excitation, primarily the ${ }^{5} \mathrm{D}_{0} \rightarrow{ }^{7} \mathrm{~F}_{2}$ and ${ }^{5} \mathrm{D}_{0} \rightarrow{ }^{7} \mathrm{~F}_{1}$ transitions in the spectral range of 603-635 and 580-603 nm, respectively [see Fig. 1(a)] [7,29].

For a plane wave in free space the magnetic field strength is related to the electric field strength by the speed of light $(B=E / c)$. With an azimuthally polarized laser beam we can drastically increase the ratio of $B / E$. In the center of the beam the electric field is zero and hence $B / E \rightarrow \infty$. However, the signal-to-noise ratio in measurements also depends on the ratio of $\max [B]$ and $\max [E]$ evaluated in different spatial locations. While the magnetic field is maximum in the center of the beam, the electric field finds its maximum at the outer ring surrounding the focal point (cf. Fig. 2). In Fig. 3(b) we have evaluated the ratio of maximum field intensities as a function of focusing angle and find that with a NA of 1.3, the ratio of maximum magnetic and electric field intensities can be enhanced by a factor of 3.4 compared to a plane wave in free space. This implies an intensity ratio of $c^{2} \max \{|B|\}^{2} / \max \{|E|\}^{2}=$ 11.6 inside the nanoparticle (with $n=1.94$ at $530 \mathrm{~nm}$ ).

In Fig. 4, we show representative excitation rate images of an $\mathrm{Eu}^{3+}$ nanoparticle (bottom row) recorded at two different excitation wavelengths. For comparison, we also include typical excitation rate images of dye-doped polystyrene nanoparticles (fluospheres). The absorption spectrum of the fluospheres is dominated by ED transitions; that is, their excitation rate scales with the local electric field intensity. Therefore, the image formed by raster scanning a fluosphere through the focus shows a doughnut shape, reflecting the electric field intensity distribution in the focus of an azimuthally polarized beam, as shown in Figs. 4(a) and 4(c). The linecuts in Figs. 4(b) and 4(d) clearly show that the intensity in the center exhibits a minimum. Because of the finite size of the fluospheres, the intensity is not exactly zero at the center.

Single $\mathrm{Eu}^{3+}$ nanoparticles, however, show a markedly different behavior. Depending on whether the excitation wavelength is tuned into resonance with a MD or ED transition, an $\mathrm{Eu}^{3+}$ nanoparticle will map out the distribution of the magnetic or the electric field. At $532.0 \mathrm{~nm}$ excitation wavelength, the $\mathrm{Eu}^{3+}$ exhibits an ED transition corresponding to one of the ${ }^{7} \mathrm{~F}_{1} \rightarrow{ }^{5} \mathrm{D}_{1}$ transitions 
[see Fig. 1(a)] [21], and hence an image taken with an azimuthally polarized beam tuned to $532.0 \mathrm{~nm}$ yields a doughnut-shaped intensity distribution with a characteristic minimum in the center, as shown in Fig. 4(h). The finite size of the nanoparticle, as well as the nearby MD transitions, prevents the intensity in the center from reaching zero. Tuning the excitation wavelength from 532.0 to $527.5 \mathrm{~nm}$ brings the $\mathrm{Eu}^{3+}$ nanoparticle into resonance with the ${ }^{7} \mathrm{~F}_{0} \rightarrow{ }^{5} \mathrm{D}_{1}$ transition [21]. Excitation with an azimuthally polarized beam gives rise to images with a circular bright spot in the center [Fig. 4(e)]. As shown in the linecut in Fig. 4(f), the intensity in the center is now a maximum, demonstrating that the particle responds primarily to the magnetic field at this excitation wavelength. Thus, by tuning a mere $5 \mathrm{~nm}$ in excitation wavelength, we can selectively excite either ED or MD transitions. Vice versa, using these transitions we can map out either local electric field distributions or local magnetic field distributions. We find that the maximum MD excitation rate in Fig. 4(f) is roughly 3.25 times larger than the maximum ED excitation rate in Fig. 4(h). Combining this information with the $c^{2} \max |B|^{2} / \max |E|^{2}$ ratio from earlier and a previously published value of the oscillator strength of the ${ }^{7} \mathrm{~F}_{0} \rightarrow{ }^{5} \mathrm{D}_{1}$ transition, we can infer the value of the ${ }^{7} \mathrm{~F}_{1} \rightarrow{ }^{5} \mathrm{D}_{1}$ oscillator strength to be $0.63 \times 10{ }^{6}$ in our nanocrystals. For details of this estimate, see the Supplemental Material [30]. We emphasize that while spontaneous MD emission from rare earth ions has been shown before [1,7-10], our work unambiguously demonstrates the selective excitation of a MD transition. Since excitation is a stimulated process, similar measurement techniques can be employed for demonstrating stimulated transitions from the excited state and achieving lasing through magnetic dipole transitions.

In the future, we hope to employ our technique to quantitatively measuring $\mathrm{MD}$ transitions in other $\mathrm{Ln}^{3+}$ lanthanides. For instance, it is known that the ${ }^{5} \mathrm{D}_{4} \rightarrow{ }^{7} \mathrm{~F}_{5}$ transition in $\mathrm{Tb}^{3+}$ has a significant MD contribution, but to date there is no precise measurement or estimate. Since the relative strength between the on-axis magnetic field and the off-axis electric field of an azimuthally polarized beam is known (cf. Fig. 3), we can, in principle, calibrate our method to measure the relative MD contribution in different $\mathrm{Ln}^{3+}$ transitions for which the precise MD proportion is unknown.

There are, however, a few limitations to our technique. First of all, it requires a high doping concentration to ensure the particles have no preferred dipole direction. Also, although many host materials (including various glasses [35] and crystals [36]) have been used for bulk samples, making highly doped stable nanoparticles from the same materials could prove challenging. Finally, to map out general magnetic field distributions, we require more effective suppression of residual ED transitions. This can be achieved, for example, by cryogenically reducing the population of the ${ }^{7} \mathrm{~F}_{1}$ state in $\mathrm{Eu}^{3+}$ or by considering quantum emitters that have a larger spectral separation between ED and MD transitions.

In conclusion, we have shown that MD transitions in $\mathrm{Eu}^{3+}$ ions can be excited optically and can therefore be used to map local magnetic fields. Future work will focus on developing scanning probes with $\mathrm{Y}_{2} \mathrm{O}_{3}: \mathrm{Eu}^{3+}$ particles attached for magnetometry applications. Recently published work has already shown the possibility of using $\mathrm{Eu}^{3+}$ particles as local probes when attached to the ends of AFM tips [29]. Using $\mathrm{Eu}^{3+}$ nanoparticles on AFM probes could potentially enable the direct mapping of magnetic fields at specific wavelengths. $\mathrm{Eu}^{3+}$ and other rare earth ions exhibit many other MD transitions in the visible region, which opens the possibility of mapping magnetic fields at multiple frequencies in parallel and furthering our understanding of light-matter interactions in inhomogeneous environments.

The authors acknowledge Palash Bharadwaj for many fruitful discussions. L. N. thanks the Swiss National Science Foundation (SNF) for financial support through Grant No. 200021 149433. L. D. C. and D. A. thank Fundação para a Ciència e a Tecnologia (Portugal), EU/ FEDER and COMPETE, Contract No. PEST-C/CTM/ LA0011/2013.

*www.photonics.ethz.ch

[1] T. Taminiau, S. Karaveli, N. van Hulst, and R. Zia, Nat. Commun. 3, 979 (2012).

[2] H. Giessen and R. Vogelgesang, Science 326, 529 (2009).

[3] M. Burresi, D. van Oosten, T. Kampfrath, H. Schoenmaker, R. Heideman, A. Leinse, and L. Kuipers, Science 326, 550 (2009).

[4] V. M. Shalaev, Nat. Photonics 1, 41 (2007).

[5] M. Burresi, T. Kampfrath, D. van Oosten, J. C. Prangsma, B. S. Song, S. Noda, and L. Kuipers, Phys. Rev. Lett. 105, 123901 (2010).

[6] S. Vignolini, F. Intonti, F. Riboli, L. Balet, L. H. Li, M. Francardi, A. Gerardino, A. Fiore, D. S. Wiersma, and M. Gurioli, Phys. Rev. Lett. 105, 123902 (2010).

[7] S. Karaveli and R. Zia, Phys. Rev. Lett. 106, 193004 (2011).

[8] J. Heber, K. Hellwege, U. Köbler, and H. Murmann, Z. Phys. 237, 189 (1970).

[9] A. Parchur and R. Ningthoujam, R. Soc. Chem. Adv. 2, 10859 (2012).

[10] J. J. D. Axe, J. Chem. Phys. 39, 1154 (1963).

[11] R. E. Kunz and W. Lukosz, Phys. Rev. B 21, 4814 (1980).

[12] T. Watrous Kelley, A. Diaz, and T. Dang, Chem. Mater. 18, 3130 (2006).

[13] G. L. J. A. Rikken and Y. A. R. R. Kessener, Phys. Rev. Lett. 74, 880 (1995).

[14] R. A. S. Ferreira, M. Nolasco, A. C. Roma, R. L. Longo, O. L. Malta, and L. D. Carlos, Chem. Eur. J. 18, 12130 (2012).

[15] U. Köbler, Z. Phys. 250, 217 (1972).

[16] G. Mhlongo, M. Dhlamini, H. Swart, O. Ntwaeaborwa, and K. Hillie, Opt. Mater. 33, 1495 (2011). 
[17] L. Ozawa, H. Forest, P. Jaffe, and G. Ban, J. Electrochem. Soc. 118, 482 (1971).

[18] H. Peng, H. Song, B. Chen, S. Lu, and S. Huang, Chem. Phys. Lett. 370, 485 (2003).

[19] D. Williams, B. Bihari, and B. Tissue, J. Phys. Chem. B 102, 916 (1998).

[20] D. Williams, H. Yuan, and B. Tissue, J. Lumin. 83 84, 297 (1999).

[21] B. Bihari, H. Eilers, and B. Tissue, J. Lumin. 75, 1 (1997).

[22] Z. Fu, S. Zhou, T. Pan, and S. Zhang, J. Lumin. 124, 213 (2007).

[23] A. Bartko, L. Peyser, R. Dickson, A. Mehta, T. Thundat, R. Bhargava, and M. Barnes, Chem. Phys. Lett. 358, 459 (2002).

[24] O. Deutschbein, Ann. Phys. (Berlin) 428, 183 (1939).

[25] J. R. Zurita Sánchez and L. Novotny, J. Opt. Soc. Am. B 19, 2722 (2002).

[26] K. S. Youngworth and T. G. Brown, Opt. Express 7, 77 (2000).

[27] Q. Zhan, Adv. Opt. Photonics 1, 1 (2009).

[28] G. S. Gowd, M. K. Patra, S. Songara, A. Shukla, M. Mathew, S. R. Vadera, and N. Kumar, J. Lumin. 132, 2023 (2012).

[29] L. Aigouy, A. Cazé, P. Gredin, M. Mortier, and R. Carminati, Phys. Rev. Lett. 113, 076101 (2014).
[30] See Supplemental Material at http://link.aps.org/ supplemental/10.1103/PhysRevLett.114.163903, which in cludes Refs. [31 34], for the calculation of the relative strengths of ED and MD transitions and for the derivation of the ${ }^{7} \mathrm{~F}_{1} \rightarrow{ }^{5} \mathrm{D}_{1}$ ED oscillator strength.

[31] M. C. E. Huber and R. J. Sandeman, Rep. Prog. Phys. 49, 397 (1986).

[32] C. Görller and K. Binnemans, in Spectral Intensities of $f f$ Transitions, edited by K. A. Gschneidner, Jr. and L. Eyring, Handbook on the Physics and Chemistry of Rare Earths Vol. 25, (Elsevier Science, Amsterdam, 1998).

[33] C. M. Dodson and R. Zia, Phys. Rev. B 86, 125102 (2012).

[34] C. K. Jørgensen and B. R. Judd, Mol. Phys. 8, 281 (1964).

[35] S. A. Saleem, B. C. Jamalaiah, A. Mohan Babu, K. Pavani, and L. Rama Moorthy, Journal of rare earths / Chinese Society of Rare Earths 28, 189 (2010).

[36] P. A. Loiko, V. I. Dashkevich, S. N. Bagaev, V. A. Orlovich, A. S. Yasukevich, K. V. Yumashev, N. V. Kuleshov, E. B. Dunina, A. A. Kornienko, S. M. Vatnik, and A. A. Pavlyuk, Laser Phys. 23, 105811 (2013). 